Supporting Information for

\title{
Impact of Shock-induced Cavitation Bubble Collapse on the Damage of Cell Membranes with Different Lipid Peroxidation Levels
}

Tong Wei, ${ }^{\dagger,}$ Lingzhi Gu, ${ }^{\ddagger}$ Mi Zhou, ${ }^{\ddagger}$ Yang Zhou, ${ }^{\ddagger} *$ Hong Yang, ${ }^{\ddagger}$ and Ming $L i^{\sharp}$

${ }^{\dagger}$ CAS Key Laboratory of Mechanical Behavior and Design of Materials, Department of Modern Mechanics, University of Science and Technology of China, Hefei 230026, China;

$\$$ Institute of Chemical Materials, China Academy of Engineering and Physics, Mianyang 621900, China. 


\section{Captions}

Table S1. Details of all simulation systems in this work.

Table S2. The bubble collapse damaging energy thresholds for membranes with different compositions

Table S3. The energy of pore formation for membranes with $0 \%$ and $100 \%$ peroxidation degree

Figure S1. Time dependence of the pressure normal to the membrane surface (pressure profile at the membrane position) with (a) $u_{p}=0.6 \mathrm{~km} \cdot \mathrm{s}^{-1}$, (b) $u_{p}=0.7$ $\mathrm{km} \cdot \mathrm{s}^{-1}$, and (c) $u_{p}=0.8 \mathrm{~km} \cdot \mathrm{s}^{-1}$ The gray area represents pressure space generated by bubble collapse

Figure S2. The comparison between the focal area of the jet and that of the local OxDOPC for the $33 \%$-membrane model in this case $u_{p}=1.0 \mathrm{~km} \cdot \mathrm{s}^{-1} . \boldsymbol{R} \mathbf{1}$ and $\boldsymbol{R} \boldsymbol{2}$ are about 7 and $22 \mathrm{~nm}$, respectively.

Figure S3. The snapshot of pore in the membrane resulting in an exchange of water molecules under shock wave $\left(u_{p}=1.0 \mathrm{~km} \cdot \mathrm{s}^{-1}\right)$.

Figure S4. A snapshot of the recovery system for OxDOPC-membrane after an equilibration time of $200 \mathrm{~ns}$ (side view).

Figure S5. Snapshots of the recovery system after an equilibration time of $200 \mathrm{~ns}$ for membranes: 0\%-membrane with bubble (a), 100\%-membrane with bubble (b, e), 0\%-membrane with no bubble (c) and 100\%-membrane with no bubble (d).

Figure S6. The water flux across the channel (black line) is shown for a recovery equilibration simulation. Here the water flux is reported as the average number of water molecules traversing the channel per ns. the number of water molecules passing through the pore per unit time using the method of Manuel et al. ${ }^{5}$

Figure S7. Log-scale representation of the out-of-plane fluctuations of the bilayers. Only $q<0.45$ values were used for fitting the $q^{4}$ behaviour ( $q$ in $\mathrm{nm}^{-1}$ unit).

Figure S8.The schematic diagram of the fixed gradient flow math model

Figure S9. The curve of ATP concentration throughout the channel with distance under fixed gradient diffusion conditions 
Appendix 1. key parameter calculation

Table S1. Details of all simulation systems in this work.

\begin{tabular}{|c|c|c|c|c|c|c|c|c|}
\hline $\begin{array}{l}\text { No. of } \\
\text { system. }\end{array}$ & $\begin{array}{c}\text { Peroxidation } \\
\text { degree } \%\end{array}$ & $\begin{array}{c}\text { Bubble } \\
\text { radius } \\
(\mathrm{nm})\end{array}$ & $\tau_{s}(\mathbf{p s})$ & $\begin{array}{c}\text { Particle } \\
\text { velocity } \\
(\mathrm{km} / \mathbf{s})\end{array}$ & $\begin{array}{c}\text { Particle } \\
\text { velocity/ } \\
\text { sound } \\
\text { velocity }\left(U_{s} / C\right)\end{array}$ & $\begin{array}{c}\text { bubble } \\
\text { collapse } \\
\text { time } \\
\text { (ps) }\end{array}$ & $\begin{array}{c}\text { Maximum } \\
\text { velocity of } \\
\text { the nanojet } \\
\left(\mathrm{km} \cdot \mathrm{s}^{-1}\right)\end{array}$ & $\begin{array}{l}\text { Maximum } \\
\text { area of } \\
\text { pore }\left(\mathrm{nm}^{2}\right)\end{array}$ \\
\hline 1 & 0 & 20 & 5 & 0.5 & 1.62 & 78 & 1.14 & 0 \\
\hline 2 & 0 & 20 & 5 & 0.6 & 1.73 & 35 & 1.55 & 0 \\
\hline 3 & 0 & 20 & 5 & 0.7 & 1.87 & 27 & 1.98 & 0 \\
\hline 4 & 0 & 20 & 5 & 0.75 & 2.00 & 24 & 2.27 & 0 \\
\hline 5 & 0 & 20 & 5 & 0.8 & 2.13 & 22 & 2.59 & 5.75 \\
\hline 6 & 0 & 20 & 5 & 0.9 & 2.27 & 20 & 3.20 & 48.75 \\
\hline 7 & 0 & 20 & 5 & 1 & 2.45 & 16 & 3.47 & 280 \\
\hline 8 & 0 & 20 & 5 & 1.3 & 2.67 & 13 & 4.46 & 338.5 \\
\hline 9 & 0 & 20 & 5 & 1.5 & 3.08 & 11 & 4.83 & 284.25 \\
\hline 10 & 0 & 0 & 5 & 1.5 & 3.08 & 0 & 0 & 0 \\
\hline 11 & 50_local & 20 & 5 & 0.5 & 1.62 & 78 & 1.14 & 0 \\
\hline 12 & 50_local & 20 & 5 & 0.6 & 1.73 & 35 & 1.55 & 0 \\
\hline 13 & 50_local & 20 & 5 & 0.7 & 1.87 & 27 & 1.98 & 0.5 \\
\hline 14 & 50_local & 20 & 5 & 0.75 & 2.00 & 24 & 2.27 & 10.5 \\
\hline 15 & 50_local & 20 & 5 & 0.8 & 2.13 & 22 & 2.59 & 125.5 \\
\hline 16 & 50_local & 20 & 5 & 0.9 & 2.27 & 20 & 3.20 & 236.5 \\
\hline 17 & 50_local & 20 & 5 & 1 & 2.45 & 16 & 3.47 & 322.5 \\
\hline 18 & 50_local & 20 & 5 & 1.3 & 2.67 & 13 & 4.46 & 298 \\
\hline 19 & 50_local & 20 & 5 & 1.5 & 3.08 & 11 & 4.83 & 256.5 \\
\hline 20 & 50_local & 0 & 5 & 1.5 & 3.08 & 0 & 0 & 0 \\
\hline 21 & 50_average & 20 & 5 & 0.5 & 1.62 & 78 & 1.14 & 0 \\
\hline 22 & 50_average & 20 & 5 & 0.6 & 1.73 & 35 & 1.55 & 0 \\
\hline 23 & 50_average & 20 & 5 & 0.7 & 1.87 & 27 & 1.98 & 0 \\
\hline 24 & 50_average & 20 & 5 & 0.75 & 2.00 & 24 & 2.27 & 0 \\
\hline 25 & 50_average & 20 & 5 & 0.8 & 2.13 & 22 & 2.59 & 1.75 \\
\hline 26 & 50_average & 20 & 5 & 0.9 & 2.27 & 20 & 3.20 & 29.75 \\
\hline 27 & 50_average & 20 & 5 & 1 & 2.45 & 16 & 3.47 & 246.25 \\
\hline 28 & 50_average & 20 & 5 & 1.3 & 2.67 & 13 & 4.46 & 329.75 \\
\hline 29 & 50_average & 20 & 5 & 1.5 & 3.08 & 11 & 4.83 & 282.25 \\
\hline 30 & 50_average & 0 & 5 & 1.5 & 3.08 & 0 & 0 & 0 \\
\hline 31 & 100 & 20 & 5 & 0.5 & 1.62 & 78 & 1.14 & 0 \\
\hline 32 & 100 & 20 & 5 & 0.6 & 1.73 & 35 & 1.55 & 0 \\
\hline 33 & 100 & 20 & 5 & 0.7 & 1.87 & 27 & 1.98 & 1 \\
\hline 34 & 100 & 20 & 5 & 0.75 & 2.00 & 24 & 2.27 & 6 \\
\hline 35 & 100 & 20 & 5 & 0.8 & 2.13 & 22 & 2.59 & 68.5 \\
\hline 36 & 100 & 20 & 5 & 0.9 & 2.27 & 20 & 3.20 & 193.25 \\
\hline
\end{tabular}




\begin{tabular}{|c|c|c|c|c|c|c|c|c|}
\hline 37 & 100 & 20 & 5 & 1 & 2.45 & 16 & 3.47 & 352.25 \\
\hline 38 & 100 & 20 & 5 & 1.3 & 2.67 & 13 & 4.46 & 292.25 \\
\hline 39 & 100 & 20 & 5 & 1.5 & 3.08 & 11 & 4.83 & 225.75 \\
\hline 40 & 100 & 20 & 10 & 0.5 & 1.62 & 25 & 2.43 & 0.25 \\
\hline 41 & 100 & 20 & 10 & 0.6 & 1.73 & 19 & 2.78 & 27 \\
\hline 42 & 100 & 20 & 10 & 0.7 & 1.87 & 16 & 3.12 & 170.25 \\
\hline 43 & 100 & 20 & 10 & 0.8 & 2.13 & 14 & 3.45 & 302.5 \\
\hline 44 & 100 & 20 & 10 & 0.9 & 2.27 & 12 & 3.70 & 348 \\
\hline 45 & 100 & 20 & 10 & 1 & 2.45 & 10 & 4.24 & 322 \\
\hline 46 & 100 & 20 & 10 & 1.3 & 2.67 & 7 & 5.50 & 236.75 \\
\hline 47 & 100 & 20 & 10 & 1.5 & 3.08 & 5 & 6.77 & 196.75 \\
\hline 48 & 100 & 10 & 5 & 0.5 & 1.62 & 30 & 1.34 & 0 \\
\hline 49 & 100 & 10 & 5 & 0.6 & 1.73 & 25 & 1.43 & 0 \\
\hline 50 & 100 & 10 & 5 & 0.7 & 1.87 & 21 & 2.17 & 0 \\
\hline 51 & 100 & 10 & 5 & 0.8 & 2.13 & 17 & 2.56 & 0 \\
\hline 52 & 100 & 10 & 5 & 0.9 & 2.27 & 16 & 3.26 & 0 \\
\hline 53 & 100 & 10 & 5 & 1 & 2.45 & 15 & 3.59 & 1.25 \\
\hline 54 & 100 & 10 & 5 & 1.3 & 2.67 & 12 & 4.25 & 26.25 \\
\hline 55 & 100 & 10 & 5 & 1.5 & 3.08 & 10 & 4.9 & 67.75 \\
\hline 56 & 100 & 10 & 10 & 0.5 & 1.62 & 17 & 2.36 & 0 \\
\hline 57 & 100 & 10 & 10 & 0.6 & 1.73 & 15 & 2.60 & 0 \\
\hline 58 & 100 & 10 & 10 & 0.7 & 1.87 & 13 & 2.81 & 0 \\
\hline 59 & 100 & 10 & 10 & 0.8 & 2.13 & 11 & 3.10 & 0 \\
\hline 60 & 100 & 10 & 10 & 0.9 & 2.27 & 10 & 3.34 & 2.5 \\
\hline 61 & 100 & 10 & 10 & 1 & 2.45 & 9 & 3.63 & 7.75 \\
\hline 62 & 100 & 10 & 10 & 1.3 & 2.67 & 6 & 4.34 & 40.5 \\
\hline 63 & 100 & 10 & 10 & 1.5 & 3.08 & 4 & 5.47 & 67.75 \\
\hline 64 & 100 & 0 & 5 & 1.5 & 3.08 & 0 & 0 & 0 \\
\hline
\end{tabular}
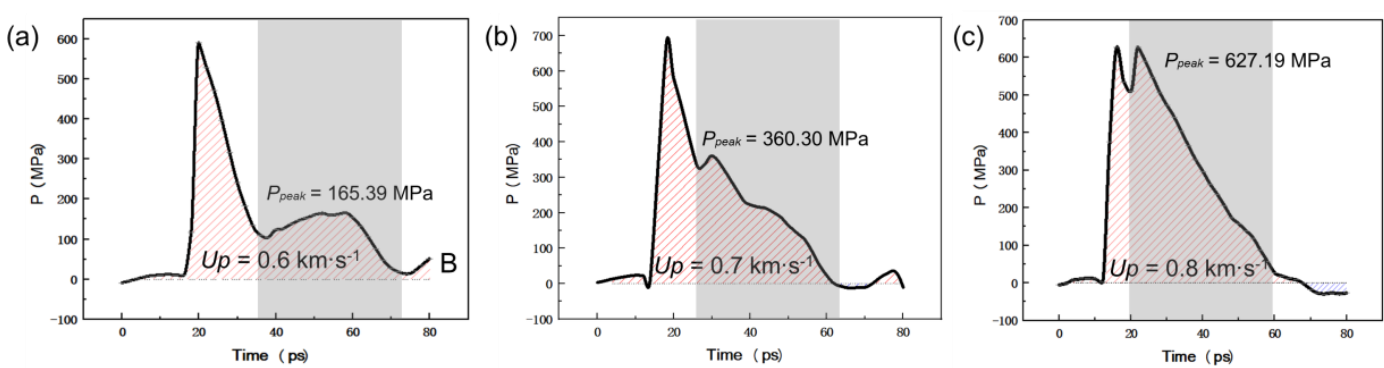

Figure S1. Time dependence of the pressure normal to the membrane surface (pressure profile at the membrane position) with (a) $u_{p}=0.6 \mathrm{~km} \cdot \mathrm{s}^{-1}$, (b) $u_{p}=0.7$ $\mathrm{km} \cdot \mathrm{s}^{-1}$, and (c) $u_{p}=0.8 \mathrm{~km} \cdot \mathrm{s}^{-1}$ The gray area represents pressure space generated by bubble collapse 


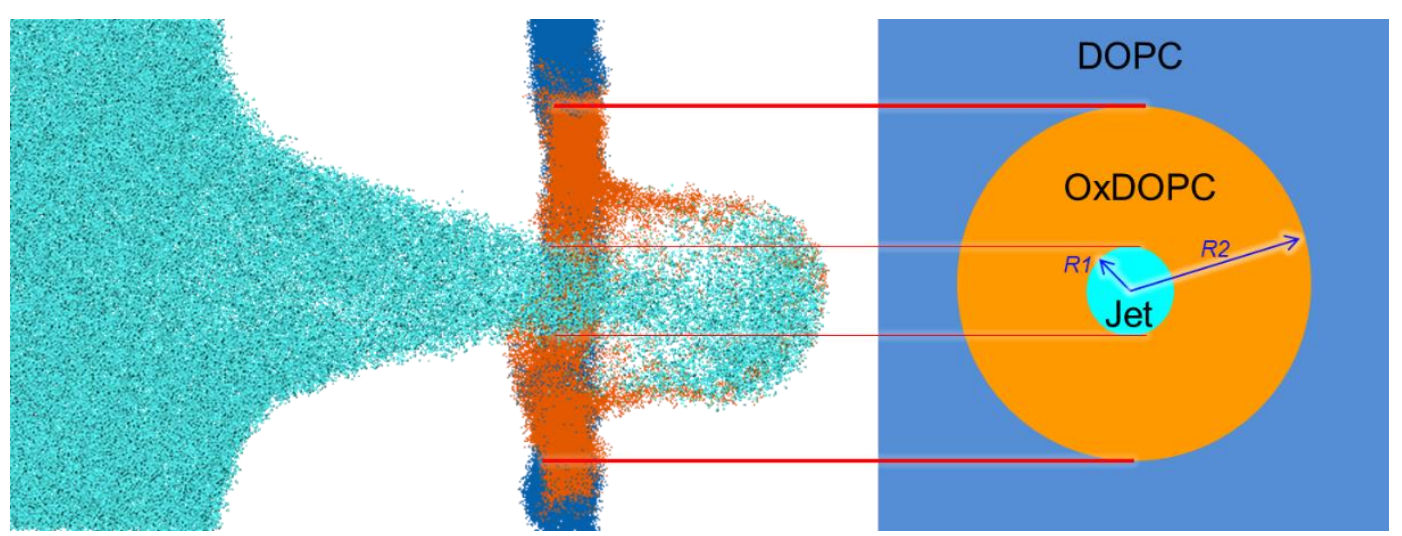

Figure S2. The comparison between the focal area of the jet and that of the local OxDOPC for the 33\%-membrane model in this case $u_{p}=1.0 \mathrm{~km} \cdot \mathrm{s}^{-1} \cdot \boldsymbol{R} \mathbf{1}$ and $\boldsymbol{R} \boldsymbol{2}$ are about 7 and $22 \mathrm{~nm}$, respectively.

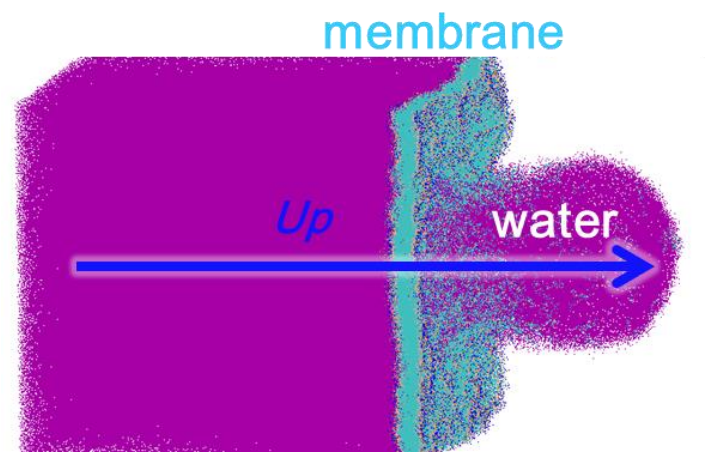

Figure S3. The snapshot of pore in the membrane resulting in an exchange of water molecules under shock wave $\left(u_{p}=1.0 \mathrm{~km} \cdot \mathrm{s}^{-1}\right)$.

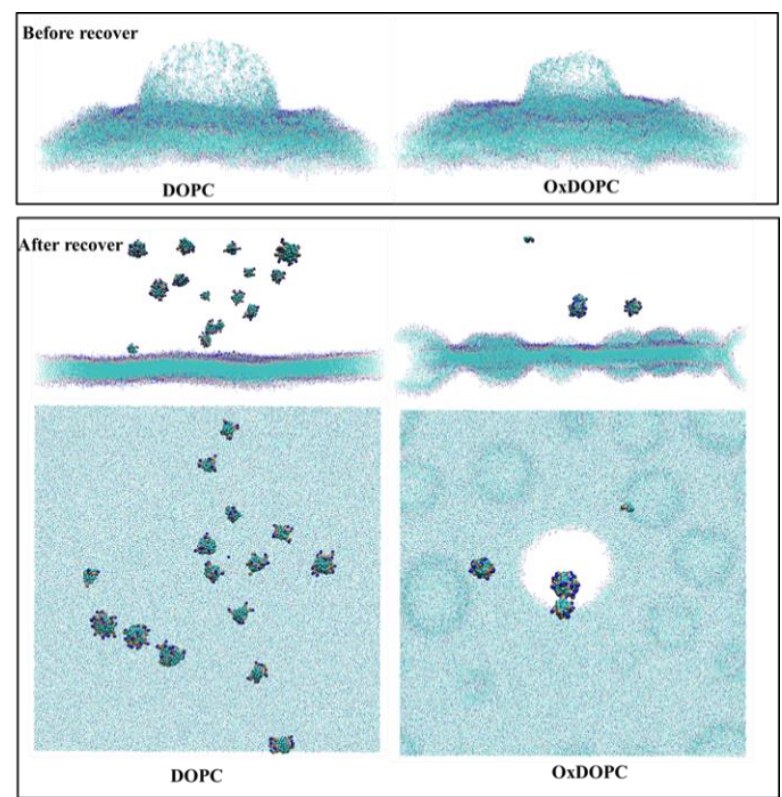

Figure S4 A snapshot of the recovery system for OxDOPC-membrane after an equilibration time of $200 \mathrm{~ns}$ (side view). 


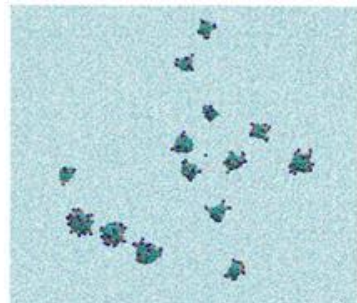

(a) bubble_0\%

(c) no bubble_0\%
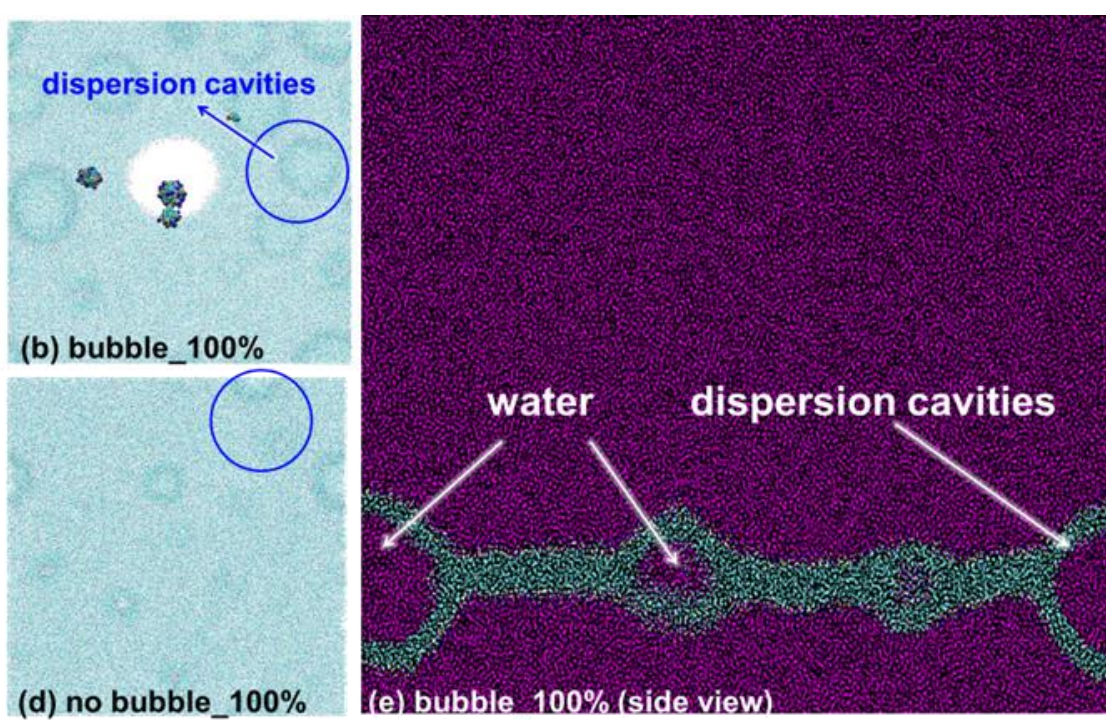

Figure S5 Snapshots of the recovery system after an equilibration time of $200 \mathrm{~ns}$ for membranes: 0\%-membrane with bubble (a), 100\%-membrane with bubble (b, e), $0 \%$-membrane with no bubble (c) and 100\%-membrane with no bubble (d).

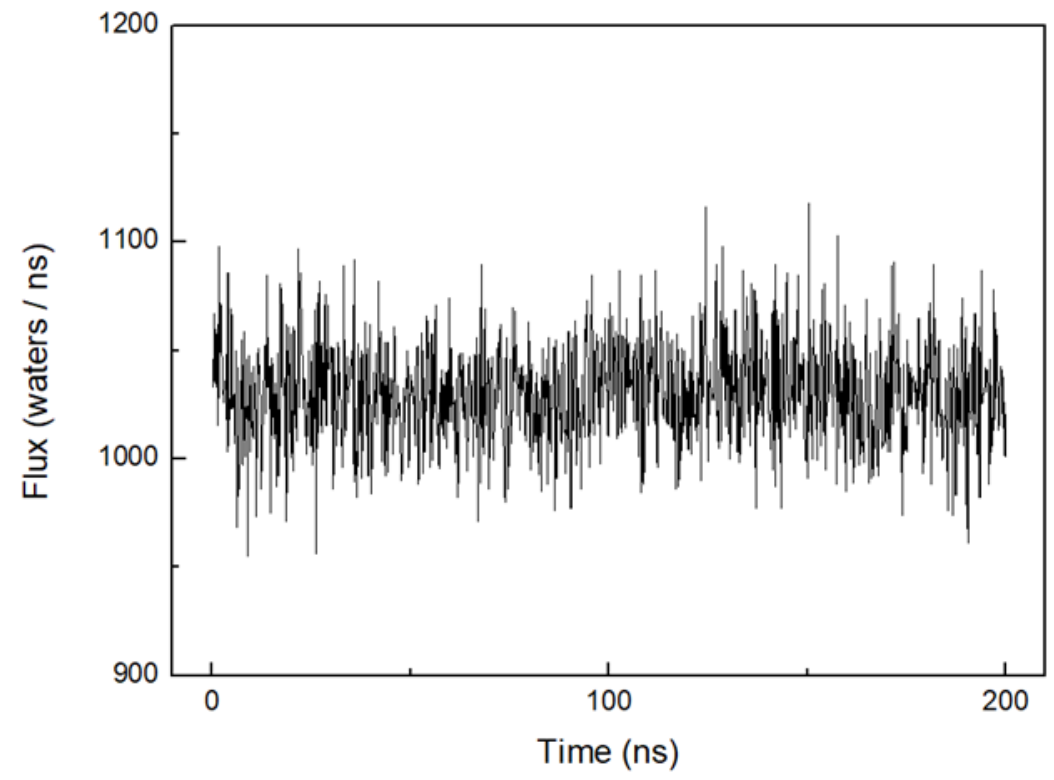

Figure S6 The water flux across the channel (black line) is shown for a recovery equilibration simulation. Here the water flux is reported as the average number of water molecules traversing the channel per ns. the number of water molecules passing through the pore per unit time using the method of Manuel et al. ${ }^{5}$

\section{Appendix 1. Key Parameter Calculation}

stretching modulus $K_{A}$

According to the following definition, the relative area $(A)$ variation consecutive to applied tension $(\sigma)$ is given by $K_{A}{ }^{1}$ : 


$$
K_{A}=\frac{1}{A}\left(\frac{d A}{d \sigma}\right)_{T, P}
$$

The elastic coefficients $K_{A}$ associated with our coarsegrained numerical models come from an area fluctuation analysis (eq (2)).

$$
K_{A}=\frac{k_{B} T\langle A\rangle}{\left\langle A^{2}\right\rangle-\langle A\rangle^{2}}=\frac{k_{B} T}{4\left(\left\langle L_{x}{ }^{2}\right\rangle-\left\langle L_{x}\right\rangle^{2}\right)}
$$

Where $\langle\mathrm{A}\rangle$ is the average value of the simple monolayer area $A=L_{x} L_{y}$, and $L_{x}$ is the average value of the lateral dimensions of the simulation box. The work of Guo et al. ${ }^{2}$ has shown that the elastic coefficient determined in this way is consistent with the one obtained by exerting a change in lateral stress (tension) on the membrane.

\section{bending modulus coefficient $\kappa_{b}$}

In the Canham-Helfrich elastic model ${ }^{3}$, the bending modulus coefficient $\kappa_{b}$ controls the out-of-plane bending deformation of the membrane. The numerical determination of the bending modulus requires a sufficiently large-scale simulation system corresponding to the small ratio $L_{b} / L_{x}$ between the membrane thickness and the lateral extension. Only under these conditions does the continuous Helfrich elastic model can reasonably describe the out-of-plane fluctuations of the membrane. Therefore, our determination of $\kappa_{b}$ is based on the equilibrium fluctuation analysis of 11520 lipid to better approach the Helfrich continuity limit. In the continuum model, the bending modulus appears in the quadratic out-of-plane fluctuation spectrum $\langle u(q) u(-q)\rangle$, as shown in eq (3):

$$
\langle u(q) u(-q)\rangle=\frac{k_{B}}{\kappa_{b}} \frac{1}{\langle A\rangle q^{4}}
$$

Where $q$ is the wave vector parallel to the membrane plane, and $u(x, y)$ the out-of-plane displacement in the Monge representation (elevation of the continuous surface).

We denote $(x i, y i, z i)$ as the space coordinates of the centre of mass of each lipid molecule in one of the bilayer leaflet, The density field in reciprocal space is given by eq $(4)^{2}$ :

$$
\hat{\rho}_{q}=\frac{1}{N_{1}} \sum_{i=1}^{N_{1}} \exp \left(i q_{x} x_{i}(t)+i q_{y} y_{i}(t)+i q_{z} z_{i}(t)\right)
$$




$$
\begin{aligned}
& =\frac{1}{N_{1}} \sum_{i=1}^{N_{1}} \exp \left(i q_{x} x_{i}(t)+i q_{y} y_{i}(t)\right)\left[1+i q_{z} z_{i}(t)\right] \\
& =\hat{\rho}_{||}(t)+i q_{z} U(q, t)
\end{aligned}
$$

Where Modes $U(q, t)$ reflect the Fourier transform of the centre of masses density field ${ }^{4}$ and $N_{l}$ as the number of lipids in this leaflet. Therefore, we simply assumed that $u\left(q_{x}, q_{y}\right) \approx U\left(q_{x}, q_{y}\right)=N_{1}^{-1} \sum_{i} z_{i} \exp \left(i q_{x} x_{i}(t)+i q_{y} y_{i}(t)\right)$ and fitted the $\ln \left(\left\langle U\left(q_{x}, q_{y}\right) U\left(-q_{x},-q_{y}\right)\right\rangle\right)$ to the straight line $C$-4ln $(q)$ (Figure S7). The intercept of the line is a function of the bending modulus.

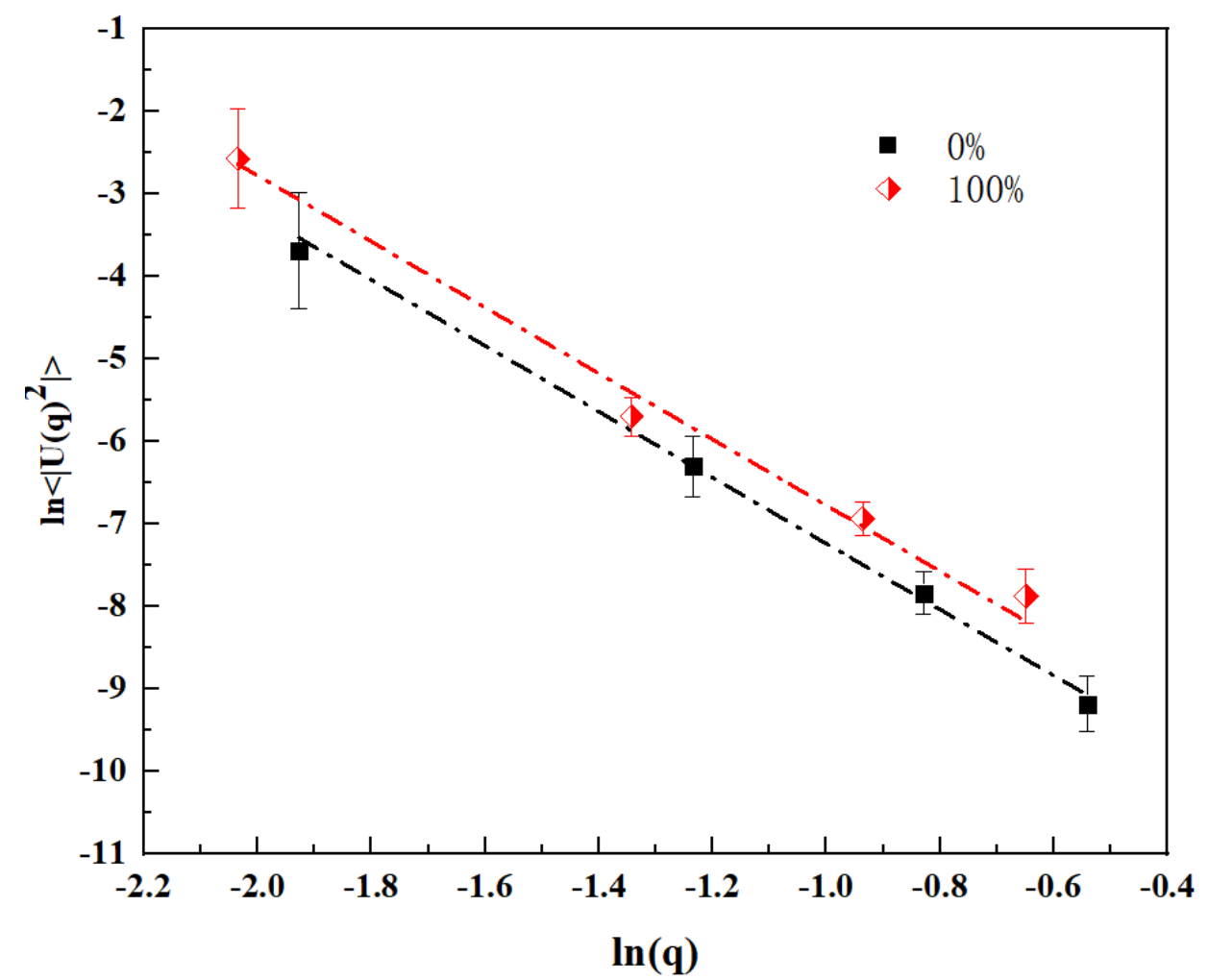

Figure S7. Log-scale representation of the out-of-plane fluctuations of the bilayers. Only $q<0.45$ values were used for fitting the $q^{4}$ behaviour ( $q$ in $\mathrm{nm}^{-1}$ unit).

\section{The total impulse $I$}

The total impulse $(I)$ is defined by eq (5):

$$
I=\int_{0}^{t_{+}} P(t) d t
$$

In the formula, $P(t)$ is the shock wave pressure and $t+$ is the time interval of the positive phase of the shock wave.

\section{The bubble collapse damaging energy thresholds}


the method of Fuad Hasan et al. ${ }^{6}$ was used to convert the damaging velocity threshold (up) of phospholipid membrane with different compositions to the threshold of energy generated by shockwave induced cavitation bubble collapses. The calculation method is shown in Equation 1:

$$
I_{c}=\frac{P_{\text {peak }}^{2}}{\rho_{L} \mathrm{C}}
$$

Where $P_{\text {peak }}$ is maximum pressure generated by bubble collapse, $\rho_{\mathrm{L}}$ is impedance, and $\mathrm{c}$ is sound velocity.

We get the maximum pressure on membrane $\left(\mathrm{P}_{\text {peak }}\right)$ as the bubble collapses from the pressure curve at the membrane during shockwave impact. As shown in Figure S1, the first wave crest is the shockwave's wave front, while the second crest is the maximum pressure generated by bubble collapse (the gray area represents pressure space generated by bubble collapse). The bubble collapse damaging energy thresholds for membranes with different compositions are shown in Table S2:

Table S2. The bubble collapse damaging energy thresholds for membranes with different compositions

\begin{tabular}{llll}
\hline Peroxidation degree & $u_{p}\left(\mathrm{~km} \cdot \mathrm{s}^{-1}\right)$ & $P_{\text {peak }}(\mathrm{MPa})$ & $I_{c}(\mathrm{MJ})$ \\
\hline $0 \%$ & 0.8 & 627.19 & 327.80 \\
$50 \%$ local & 0.7 & 360.30 & 123.63 \\
$50 \%$ Average & 0.8 & 627.19 & 327.80 \\
$100 \%$ & 0.7 & 360.30 & 123.63 \\
\hline
\end{tabular}

\section{The gradient diffusion of ATP}

We established a fixed gradient flow math model to calculate the gradient diffusion of ATP and other molecules leaked from cells. The schematic diagram of the mathematical model is shown in Figure S8. This model mainly relies on two basic equations: one is Fick's Law, and the other is Solute Mass Conservation Equation. 


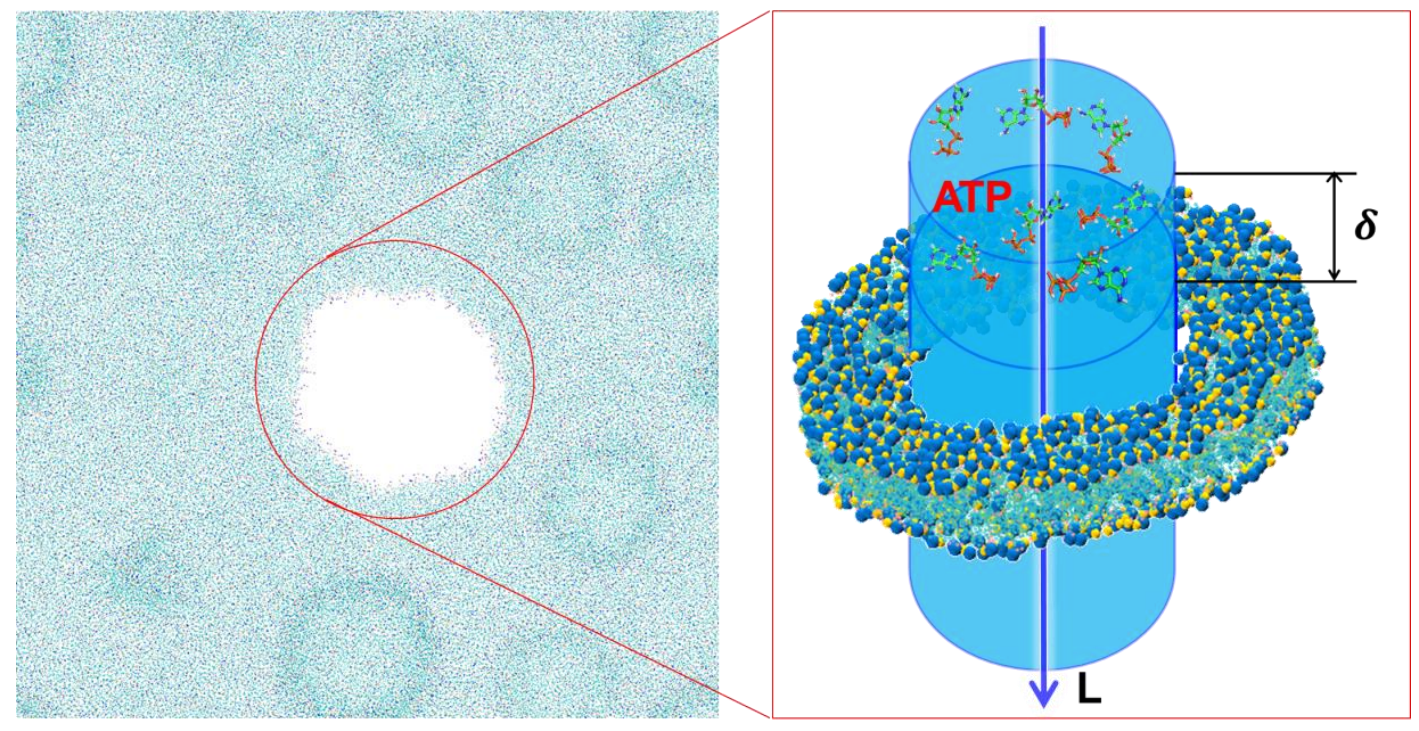

Figure S8. The schematic diagram of the fixed gradient flow math model

Firstly, we define the section of cylinder flow passing through the pore as the experiment cylinder of this mathematics model. The parameters: $a$ is cross-section area of the experiment cylinder, $c$ is cycle length of the cross section, $C_{0}$ is initial concentration of ATP, $\delta$ is cylinder length inside the cell, and L is solute flow direction.

Consider an experiment cylinder from $x^{*}$ to $x^{*}+\Delta x^{*}$. Take the direction $\mathrm{x}^{*}$ increases as positive. $N^{*}\left(x^{*}\right)$ is the rate that ATP diffuses into the channel when it passes by unit area at $x^{*}$ boundary. $F^{*}\left(x^{*}\right)$ is number of ATP that pass through unit area within unit time at $x^{*}$ in the direction of $L$. The net flow passes through the experiment cylinder is zero, and we get:

$\mathrm{c} \int_{x^{*}}^{x *+\Delta x *} N^{*}(s) d s+a\left[F^{*}\left(x^{*}\right)-F^{*}\left(x^{*}+\Delta \mathrm{x}^{*}\right)\right]=0$

Using Integral Mean Value Theorem, we divide equation by $\Delta x^{*}$, and let $\Delta x^{*} \rightarrow 0$, then we get equation 2 :

$\mathrm{c} N^{*}\left(x^{*}\right)-\mathrm{a} \frac{d F^{*}}{d x^{*}}=0$

The solute flux of unit area, $F^{*}$, is the addition of two parts, which are convection flux $F_{c o n v}^{*}$ and diffusion flux $F_{\text {diff }}^{*}$. We firstly determine the contribution of 
convection. This part is induced by the transport of fluid's whole movement. If fluid at $x^{*}$ has speed $v^{*}$, fluid volume passing through unit area within unit time is $v^{*}\left(x^{*}\right)$. If $C^{*}$ is the mass of ATP in unit fluid volume, then convection flux is

$F_{\text {conv }}^{*}=C^{*} v^{*}$

According to Flick's Law, diffusion flux is

$F_{d i f f}^{*}=-D\left(\frac{d C^{*}}{d x^{*}}\right)$

So, the total flux of ATP passing through the experiment cylinder is

$F^{*}=C^{*} v^{*}-\mathrm{D} \frac{d C^{*}}{d x^{*}}$

To describe ATP pumped from intracellular region to the experiment cylinder in a simple way, we set the rate that ATP is pumped from intracellular region to the cylinder $N^{*}=D$, and in other regions $N^{*}=0$,

So $0 \leq x^{*} \leq \delta, N^{*}=D, \delta \leq x^{*} \leq L, N^{*}=0$

Join (2) and (6), and integrate:

$\mathrm{a} F^{*}=c D x^{*}+Q_{1}, 0 \leq x^{*} \leq \delta$

$\mathrm{a} F^{*}=Q_{2}, \delta<x^{*} \leq \mathrm{L}$

$F^{*}(0)=0$, we get $Q_{1}=0$. Due to the continuity of ATP concentration, $C^{*}$ and $F^{*}$ are continuous at $\delta, Q_{2}=c D \delta$.

We get mathematical description of ATP's diffusion under fixed gradient:

$C^{*} v^{*}-\mathrm{D} \frac{d C^{*}}{d x^{*}}=\left\{\begin{array}{cc}a^{-1} c D x^{*}, & 0 \leq x^{*} \leq \delta \\ a^{-1} c D \delta, & \delta<x^{*} \leq \mathrm{L}\end{array}\right.$

The boundary conditions are $v^{*}(0)=0, C^{*}(0)=C_{0}$

It seems difficult to directly estimate the value of $v^{*}$ in the equation. However, as the flow is driven by ATP concentration gradient, the diffusion effect cannot exceed convection effect even in the most extreme conditions:

$F^{*}(\mathrm{~L}) \geq v^{*}\left(x^{*}\right) C^{*}(\mathrm{~L})$

$v^{*}(\mathrm{~L}) \leq \frac{c D \delta}{2 a C_{0}}$

Setting diffusion effect as $1 / 2$ of convection effect, i.e. $v^{*}(\mathrm{~L})=\frac{c D \delta}{2 a c_{0}}$, and we get general solution to the differential equation: 
$C^{*}=\frac{1}{2} C_{0} e^{-\frac{c \delta}{a C_{0}} x^{*}}+\frac{1}{2} C_{0}$

From work of Niki et al. ${ }^{7}$ we can obtain the initial concentration $\left(C_{0}\right)$ of ATP in the cell. Finally, we can get a curve reflecting the change of ATP concentration throughout the channel with distance under fixed gradient diffusion conditions (Figure S9).

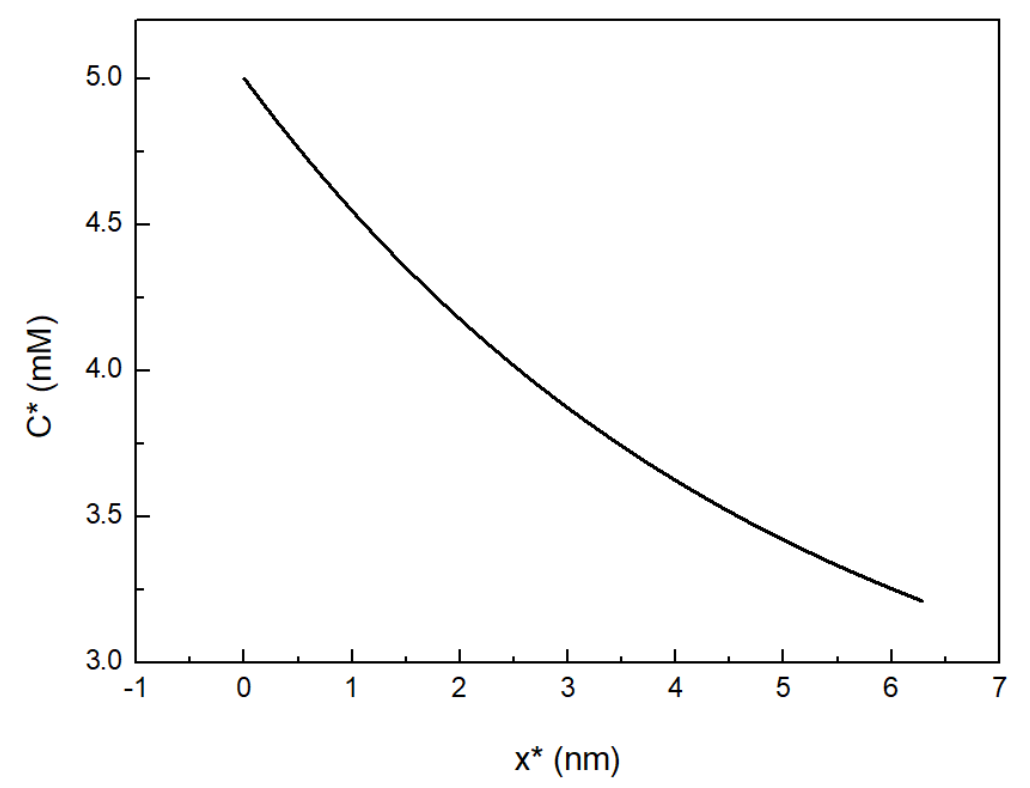

Figure S9. The curve of ATP concentration throughout the channel with distance under fixed gradient diffusion conditions

\section{The energy of pore formation}

We calculate the energy of pore according to the work of Nelson et al (equation 1). ${ }^{8,9}$

$$
E=2 \pi r a+\pi r^{2} b
$$

where $\mathrm{a}=2 K_{A}\left(\frac{\varepsilon_{c}^{3} A_{0}}{27 \pi}\right)^{\frac{1}{2}}, r$ is the pore radius and the lateral stretching area strain $\varepsilon_{c}=$ $A_{\|} /\left(A_{0}-1\right)$. In NVE ensemble, $\varepsilon_{c}$ is 0 (no area variation), that is, a=0. Finally, the energy of pores formation during shock only couples with surface tension $(b=$ $\left.I_{z}\left[P_{z}-\frac{P_{x}+P_{y}}{2}\right]\right)$ of membrane and the pore radius, i.e., $E=\pi r^{2} b$. The results in Table S3 show that comparing the $0 \%$-membrane, the $100 \%$-membrane has a smaller surface critical tension and formats a pore with a larger radius.

Table S3. The energy of pore formation for membranes with $0 \%$ and $100 \%$ 
peroxidation degree

\begin{tabular}{lllll}
\hline Peroxidation degree & $u_{p}\left(\mathrm{~km} \cdot \mathrm{s}^{-1}\right)$ & $r(\mathrm{~nm})$ & $b(\mathrm{mN} / \mathrm{m})$ & $E\left(10^{-17} \mathrm{~J}\right)$ \\
\hline $0 \%$ & 0.7 & 3.94 & 408.65 & 2.12 \\
$100 \%$ & 0.7 & 7.85 & 117.14 & 2.26 \\
\hline
\end{tabular}

\section{- REFERENCES}

(1) Devaux; P., Phospholipid bilayers: Physical principles and models: by G. Cevc and D. Marsh. John Wiley \& Sons, Chichester, 1987, pp. 442, 73.35. Biochimie 1988, 70, 1121-1121.

(2) YachongGuo; Baulin, V. A.; FabriceThalmann, Peroxidised phospholipid bilayers: insight from coarse-grained molecular dynamics simulations. Soft Matter 12, 263-271.

(3) Frieske, H.; Mahnig, M., Elastic properties of lipid bilayers: theory and possible experiments. $Z$. Naturforsch. C: Biosci. 1973, 28, 693-703.

(4) Brandt, E. G.; Braun, A. R.; Sachs, J. N.; Nagle, J. F.; Edholm, O., Interpretation of Fluctuation Spectra in Lipid Bilayer Simulations. Biophys. J. 2011, 100, 2104-2111.

(5) Melo, M. N.; Arnarez, C.; Sikkema, H.; Kumar, N.; Walko, M.; Berendsen, H.; Kocer, A.; Marrink, S. J.; Ingólfsson, H., High-throughput simulations reveal membrane-mediated effects of alcohols on MscL gating. J. Am. Chem. Soc. 2017, 139, 2664-2671.

(6) Hasan, F.; Al Mahmud, K. A. H.; Khan, M. I.; Patil, S.; Dennis, B. H.; Adnan, A., Cavitation Induced Damage in Soft Biomaterials. Multiscale Sci Eng 2021, 3, 67-87.

(7) Niki, I.; Ashcroft, F. M.; Ashcroft, S. J., The dependence on intracellular ATP concentration of ATP-sensitive K-channels and of Na,K-ATPase in intact HIT-T15 $\beta$-cells. FEBS Lett 1989, 257, 361-364.

(8) Moroz, J. D.; Nelson, P., Dynamically-Stabilized Pores in Bilayer Membranes. Biophys J 1997, 72, 2211-2216.

(9) Zhelev, D. V.; Needham, D., . Tension-stabilized pores in giant vesicles: determination of pore size and pore line tension. Biochim Biophys Acta 1993, 1147, 89-104. 